\title{
ROLE OF ANTICONVULSANT THERAPEUTIC DRUG MONITORING IN IMPROVING CLINICAL OUTCOMES: AN EXAMPLE OF 12 ADULT EPILEPSY PATIENTS
}

Ana Rankovic ${ }^{1}$, Jasmina Milovanovic ${ }^{2}$, Snezana V Jankovic ${ }^{2}$, Natalija Todorovic ${ }^{3}$, Nemanja Rancic ${ }^{4}$, Nikola Jestrovic ${ }^{5}$, Iztok Grabnar ${ }^{6}$, Slobodan Jankovic ${ }^{2}$, Mihajlo Jakovljevic ${ }^{2}$

${ }^{1}$ Radiology Diagnostic Service, The Clinical Centre, Kragujevac, Serbia

${ }^{2}$ Pharmacology and Toxicology Department, The Medical Faculty, Kragujevac, Serbia

${ }^{3}$ The Neurology Department, The Clinical Centre, Kragujevac, Serbia

${ }^{4}$ The Medical Faculty University of Kragujevac, Serbia

${ }^{5}$ Merck Sharp \& Dohme Idea Inc., AG Beograd, Serbia

${ }^{6}$ The Faculty of Pharmacy, University of Ljubljana, Slovenia

\section{ULOGA KONTROLISANJA ANTIKONVULZIVNE TERAPIJE U UNAPREDJIVANJU KLINICKIH REZULTATA NA PRIMERU 12 ODRASLIH OBOLELIH OD EPILEPSIJE

Epilepsy, one of the most common neurological diseases, demands persistent and long-term therapy. In spite of the available therapeutic interventions for seizure disorders, the incidence of epilepsy and mortality associated with "status epilepticus" remain significant (1). The goal of seizure management is satisfying seizure control with minimal side effects (2). Quality of life (QoL) studies have suggested that patients who suffer even a single seizure per year exhibit significantly reduced QoL (3). Among the wide range of medications offered in the market, valproic acid is still considered by many to be "a gold standard" to treat many convulsive disorders, including absence, generalised tonic-clonic seizures, myoclonic juvenile seizures, and photosensitive seizures. It also exhibits an acceptable toxicity profile compared with other anticonvulsants (4).

The Serbian health care system is still not capable of systemic therapeutic drug monitoring in clinical practice. The underlying reasons are typical for an upper-middle income transitional market and are attributed to both financial constraints and the lack of skilled, highly educated human resource availability in the field. These reasons influenced the authors to report pilot trial results to provide a small move forward on the issue among local clinicians (5).

The core aim of the trial presented was an exploration of the dose-response relationship in a small group of twelve adults suffering from epilepsy. The authors studied frequencies of drug adverse effects associated with long-term monotherapy and evaluated the appropriateness and clinical value of regular therapeutic drug monitoring (TDM) of valproic acid. The presence of a correlation between drug plasma concentration as an independent variable and frequency of seizures, frequency of adverse events and overall life quality as dependent variables was also tested. The reported results are an unpublished fragment originating from a large-scale collaborative project on pharmacokinetic modelling in juvenile epilepsy treatment (3-6).

This study was performed in 2007 on a small group of 12 adult patients suffering from clinically confirmed epilepsy. An anticonvulsant drug was administered in a full dosing regimen, and steady state was achieved prior to study inclusion. These patients were clinically followed at the Department of Neurology, Clinical Centre, Kragujevac, for three months. Crosssectional analyses and the determination of clinical outcomes (adverse event frequency and life quality) at both inclusion (zero point) and the end of the study, except for the seizure frequency, were performed . All of the patients were using acid-resistant, film-coated valproate tablets with $2 / 3$ sodium valproate and $1 / 3$ valproic acid content with the brand name "Eftil", which was produced by "Zorka Pharma", Serbia. The determination of valproic acid and its salt concentrations in patient serum samples was performed by fluorescence polarisation immunoassay on an Abbott TDX commercial/FLX device using a set of reagents from the same manufacturer. Valproate was administered two to three times per day in oral form at a daily dose of 250 to $1000 \mathrm{mg}$. The patients were previously on this drug regimen for 4 to 516 months. The drug concentration in the serum was measured at two points: 2 hours after taking the morning dose (when the peak concentration in blood is expected, which is consistent with known drug kinetics) and 12 hours after ingestion (minimum trough concentration in the blood), e.g., before taking the morning dose.

The effectiveness of treatment outcome in terms of seizure control was estimated by the attending neurologist. This effectiveness was based on the frequency and severity criteria of seizure clinical appearance and regular electroencephalographic examination. A seizure diary was regularly kept, providing evidence of the occurrence, characteristics and frequency of attacks by the patient or a family member. The fact 
that most patients lose consciousness during the seizure and have no clear aura but feel recognisable postictal symptoms makes the patient records unreliable. In addition, relatively frequent nocturnal seizures often go unnoticed (7). Because stigmatisation of this disease is common among Balkan nations, it is most often taken seriously by the affected individuals and their family members. Therefore, the expected level of compliance with the treating neurologist is high. The importance of the chronic toxicity of anticonvulsants with a number of cognitive and motor side effects was recognised early (8). In addition, anticonvulsant use is a long-term daily therapy (9). We provided data on side effects through guided interviews with patients and structured questionnaires. The majority of anticonvulsive substances are liposoluble compounds with good penetration through the haematoencephalic barrier and significant impact on emotional life and cognitive processes of the patient. The main clinical outcome, which was epilepsy-related quality of life, was assessed through the QOLIE-31 questionnaire, which has been previously validated and standardised in the Serbian language.

Trial results are presented in Table 1 and Graph 1. A common linear chart shows the arithmetic means of measured values of clinical outcomes in the beginning and at the end of the trial period for each patient observed. Drug plasma concentrations are intentionally provided separately: one measurement represents the arithmetic mean of respective (first and last time point) trough values, and the other one represents the arithmetic mean of peak values per patient. The average valproic acid plasma concentration was $93.98 \pm 26.43 \mathrm{mg} / \mathrm{l}$. The average number of seizures during the three months was $25.47 \pm 93.52$. There was a wide range of drug-related adverse effects. The average number of side effects was $5.06 \pm 4.91$ per patient. The valproate concentrations measured were in accordance with expected drug pharmacokinetics and within the desired therapeutic range of 40 to $100 \mu \mathrm{g} / \mathrm{l}$ (4). These values confirm that the therapeutic dose was, in most cases, adequately prescribed and individually adjusted.
In many patients on prolonged therapy, the frequency and severity of drug side effects are roughly correlated with measured plasma concentrations (9). The consequence is obvious: a higher available fraction of the free drug leads to a higher concentration near the receptors, more extensive binding and a stronger expected biological response. This model is simplified according to what is happening in vivo. Tomson et al. obtained similar results, which showed that TDM had special importance for the new generation of antiepileptic drugs (10). Because of the narrow therapeutic range, the side effect rate was significantly higher, and therefore, performing TDM is invaluable. We noticed an absence of correlation between both peak and through plasma concentration values and the frequency of side effects. In particular, a high frequency of certain adverse events, such as disturbances related to thinking and memory, drowsiness, headache, fatigue, and increased body mass, was observed.

These cognitive- and motor skill-related adverse events are supposed to significantly contribute to quality of life decreases caused by therapy. There were no significant correlations between quality of life scores and peak or trough concentrations in patients. This result was found for both particular domains (seizure concern, overall life quality, emotional well-being, fatigueless/energy, cognitive functions, drug effects, and social activities) and an overall value. We should emphasise that our patient sample consisted mostly of people who had been diagnosed for years prior to study inclusion. There were no psychiatric comorbidities. The adolescent psychosexual personality maturation process was already complete. Most of these patients have also accepted the inevitability and peculiarity of their disease. Considering these issues, we can explain the balanced quality of life scores detected (3). However, the authors still believe that careful adjustment of the dosage regimen could lead to better life quality in patients suffering from epilepsy. To achieve this purpose, measuring plasma drug concentrations and gradually adjusting the dose are necessary.

\begin{tabular}{|c|c|c|c|c|c|}
\hline Case number & $\begin{array}{c}\text { Average } \\
\text { peak value }\end{array}$ & $\begin{array}{c}\text { Average } \\
\text { trough value }\end{array}$ & $\begin{array}{c}\text { Seizure } \\
\text { frequency }\end{array}$ & $\begin{array}{c}\text { Adverse event fre- } \\
\text { quency }\end{array}$ & $\begin{array}{c}\text { Quality of life over- } \\
\text { all score }\end{array}$ \\
\hline 1 & 98.295 & 54.14 & 0 & 1 & 82.85 \\
\hline 2 & 139.38 & 83.98 & 0 & 2.5 & 67.7 \\
\hline 3 & 120.53 & 95.035 & 14 & 12 & 59.8 \\
\hline 4 & 132.325 & 125.49 & 1 & 1 & 48.95 \\
\hline 5 & 112.92 & 106.675 & 0 & 1 & 58.05 \\
\hline 6 & 98.975 & 83.06 & 0 & 3 & 90.5 \\
\hline 7 & 89.755 & 55.785 & 58 & 3 & 54.6 \\
\hline 8 & 86.83 & 58.145 & 3 & 9.5 & 36.75 \\
\hline 9 & 89.96 & 117.355 & 0 & 5 & 75.45 \\
\hline 10 & 69.85 & 54.865 & 0 & 4 & 55.25 \\
\hline 11 & 58.03 & 67.975 & 3 & 11.5 & 67.65 \\
\hline 12 & 54.9 & 59.425 & 48 & & 54.8 \\
\hline
\end{tabular}

Table 1. Arithmetic means of plasma concentrations and related clinical outcome values measured at the beginning and the end of clinical follow-up 
Graph 1. Arithmetic means of plasma concentrations and related clinical outcome values measured at the beginning and the end of clinical follow-up

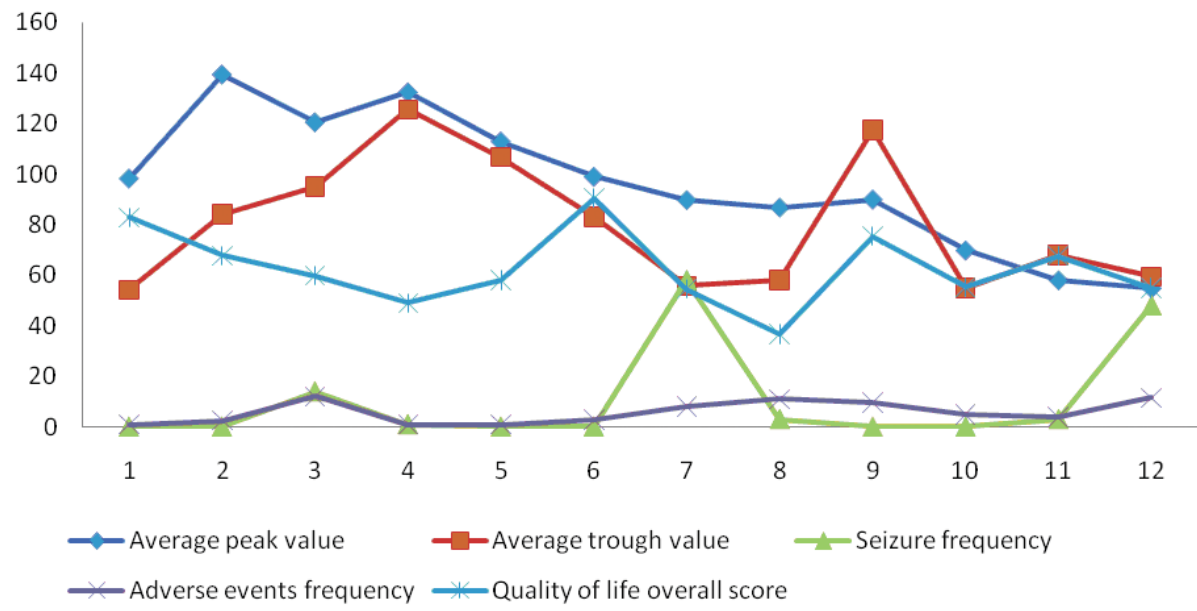

No significant correlation was observed between seizure frequency and trough or peak concentrations of valproate. Patients were in an equilibrium state of drug in the body prior to study inclusion. During that time, the choice of drug, time and route of application and the dosage regimen were carefully titrated according to the feedback on clinical effectiveness. Therefore, the absence of such a correlation can partly be interpreted by previously established satisfactory seizure control in many patients (8). Other causes could be natural gradual improvement of most juvenile epilepsies towards the third decade of life and the lack of appropriate sample size availability associated with high co-medication rates of adults in this study.

Because TDM was envisaged in seizure disorder treatment guidelines for a number of years, systematic reviews of decent methodological quality have been written on this topic, with opposing views (10). One of the main practical recommendations from our experiences with the pharmacokinetics of antiepileptic drugs is the important place and utility of TDM procedures. Based on our results, it appears that TDM of valproic acid and its salts is fully justified for optimising the dosage regimen. If these findings were proved in a larger pool of patients, it would mean that by adjusting drug concentration in the blood, we can significantly shape the main target of epilepsy treatment, quality of life. Although among our research population sample, the assumed dose-response relationship was weak, we certainly recommend further pharmacokinetic explorations of this type. More individually adjusted dosing regimens would certainly provide more satisfactory longterm clinical outcomes.

\section{ACKNOWLEDGEMENTS}

The authors would like to express their gratitude to the Medical Faculty University of Kragujevac for Internal Scientific Research Grants numbers 17/10 and 08/08, the Ministry of Science and Technological Development of the Republic of Serbia for Grant $\mathrm{N}^{\circ} 175014$, and European Programs for Education and Training - CEEPUS Mobility Grant Program (Contract No. 2006-4385-CII-0607-8000), through which this research project was jointly financed.

\section{REFERENCES}

1. Marchi N, Granata T, Freri E, Ciusani E, Ragona F, Puvenna V, Teng Q, Alexopolous A, Janigro D. Efficacy of anti-inflammatory therapy in a model of acute seizures and in a population of pediatric drug resistant epileptics. PLoS One 2011; 6(3): s18200.

2. Kwan P, Arzimanoglou A, Berg AT, Brodie MJ, Allen Hauser W, Mathern G, Moshé SL, Perucca E, Wiebe S, French J. Definition of drug resistant epilepsy: consensus proposal by the ad hoc Task Force of the ILAE Commission on Therapeutic Strategies. Epilepsia 2010; 51(6): 1069-77.

3. Jakovljevic MB, Jankovic SM, Jankovic SV, Todorovic $\mathrm{N}$. Inverse correlation of valproic acid serum concentrations and quality of life in adolescents with epilepsy. Epilepsy Res 2008; 80(2-3): 180-3.

4. Jakovljević MB, Janković SM, Todorović N, Milovanović JR, Janković S. Pharmacokinetic modelling of valproate in epileptic patients. Med Pregl 2010; 63(5-6): 349-55.

5. Jakovljevic M, Jovanovic M, Lazic Z, Jakovljevic V, Djukic A, Velickovic R, Antunovic M. Current efforts and proposals to reduce healthcare costs in Serbia, Ser J Exp Clin Res 2011; 12 (4): 161-163.

6. Vovk T, Jakovljević MB, Kos MK, Janković SM, Mrhar A, Grabnar I. A nonlinear mixed effects modelling analysis of topiramate pharmacokinetics in patients with epilepsy. Biol Pharm Bull 2010; 33(7): 1176-82.

7. The National Society for Epilepsy. The Seizure diary formulary for patients, UK, 2007.

8. Wilby J, Kainth A, Hawkins N, Epstein D, McIntosh H, McDaid C, Mason A, Golder S, O'Meara S, Sculpher M, Drummond M, Forbes C. Clinical effectiveness, tolerability and cost-effectiveness of newer drugs for epilepsy in adults: a systematic review and economic evaluation. Health Technol Assess 2005; 9(15): 1-157.

9. Mei PA, Montenegro MA, Guerreiro MM, Guerreiro CA. Pharmacovigilance in epileptic patients using antiepileptic drugs. Arq Neuropsiquiatr 2006; 64(2A): 198-201.

10. Tomson T, Dahl ML, Kimland E. Therapeutic monitoring of antiepileptic drugs for epilepsy. Cochrane Database Syst Rev 2007;1: CD002216. 